\title{
Varying division planes of secondary constrictions in spheroidal Escherichia coli cells
}

\author{
Arieh Zaritsky, ${ }^{1}$ Conrad L. Woldringh, ${ }^{2}$ Itzhak Fishov, ${ }^{1}$ \\ Norbert O. E. Vischer ${ }^{2}$ and Monica Einav ${ }^{1}$
}

Author for correspondence: Arieh Zaritsky. Tel: $+97276461712 / 6278$ 951. Fax: +97276278 951/6472 890. e-mail : ariehz@bgumail.bgu.ac.il

1 Department of Life Sciences, Ben-Gurion University of the Negev, PO Box 653, Be'er-Sheva 84105 , Israel

2 Section of Molecular Cytology, Institute for Molecular Cell Biology, BioCentrum, University of Amsterdam, Kruislaan 316, 1098 SM Amsterdam, The Netherlands

\begin{abstract}
Planes of successive divisions in Escherichia coli have been proposed to be either parallel or perpendicular to each other, restricted to one or two dimensions. To test the hypothesis that divisions can occur in planes alternating in three dimensions, a method was developed to generate cells with secondary constrictions during growth in suspension. The method involves a combination of thymine limitation (to manipulate chromosome replication rate) and mecillinam treatment (to inhibit penicillin-binding protein 2). The former modifies timing of terminations, the latter results in spheroidal cells. Such cells displayed secondary constrictions after adding deoxyguanosine (accelerating replication rate), thus temporarily enhancing division signals. The successive constrictions were seen to develop in planes that were tilted relative to each other, and in positions related to those of the nucleoids, visualized by staining with DAPI (4',6-diamidino-2-phenylindole dihydrochloride hydrate). Visualizing cell envelopes with FM 4-64 by confocal scanning laser microscopy supported the conclusion that planes of successive divisions can alternate in three dimensions.
\end{abstract}

Keywords : division planes, thymine limitation, nucleoid segregation, fluorescence, electron microscopy

\section{INTRODUCTION}

To sustain a stable cell line with uniform genetic information, a mechanism must exist that couples division to chromosome replication (Mitchison, 1971; Cooper, 1991). A coupling mechanism has been envisaged because in Escherichia coli cell division occurs a constant time after termination of replication (about $20 \mathrm{~min}$ at $37^{\circ} \mathrm{C}$ ) for a wide range of doubling times obtained by various nutrient regimes (Helmstetter $\mathrm{et}$ al., 1968). This relationship is maintained even under conditions which delay termination by slowing replication velocity (Zaritsky \& Pritchard, 1973; Lane \& Denhardt, 1975). Such coupling (D'Ari \& Bouloc, 1990) can also be inferred from various mutants defective in DNA replication and partitioning despite indications for an independent pathway when oriC was replaced by plasmid R1 (Bernander \& Nordström, 1990). The coupling hypothesis is further confirmed by the rare occurrence of constrictions at sites surrounding repli-

Abbreviations: CSLM, confocal scanning laser microscopy; DAPI, 4,6diamidino-2-phenylindole dihydrochloride hydrate; SCC, secondaryconstricted cells; SEM, scanning electron microscopy. cating and segregating nucleoids (Van Helvoort et al., 1998).

Bacillary bacteria naturally grow by elongation and divide in a plane perpendicular to their long axis (Bloom et al., 1974; Woldringh et al., 1988). At faster growth rates the cells are wider, retaining an almost constant shape (defined by the mean length/diameter ratio; Zaritsky, 1975). Specific inhibition of division by low penicillin $G$ concentrations (Hadas et al., 1995) results in filaments (Donachie \& Begg, 1970), while specific inactivation of Pbp2 (Spratt, 1978) results in spheroids (Bouloc et al., 1989). The successive division planes of such agar-grown spheroidal cells seem to alternate in two perpendicular planes, forming tetrads (Donachie $e t$ al., 1995). This conclusion has recently been disputed on methodological grounds (Cooper, 1997): when cells were grown in the less viscous Methocel, subsequent divisions seemed to occur in planes that were parallel to one another. Cooper has therefore suggested that growing cells on an agar surface caused them to rotate after division, giving the appearance of subsequent divisions occurring at perpendicular planes.

In a previous attempt (C. L. Woldringh \& A. Zaritsky, 
(a) Before step-up $(C=80 \mathrm{~min})$
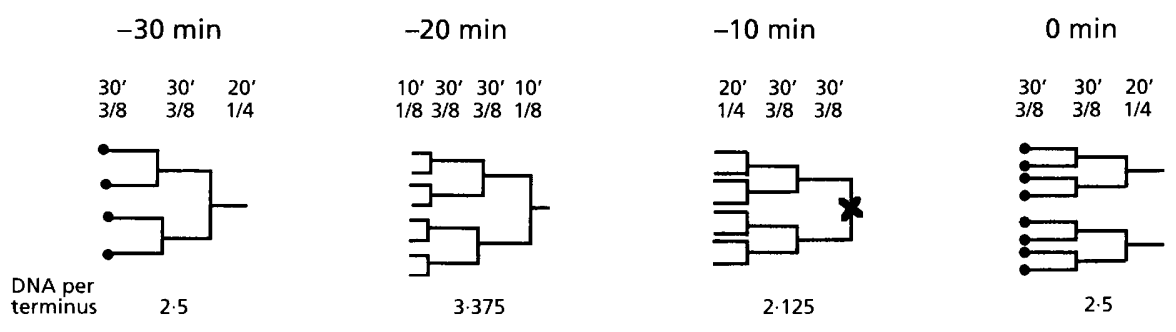

(b) During step-up transition
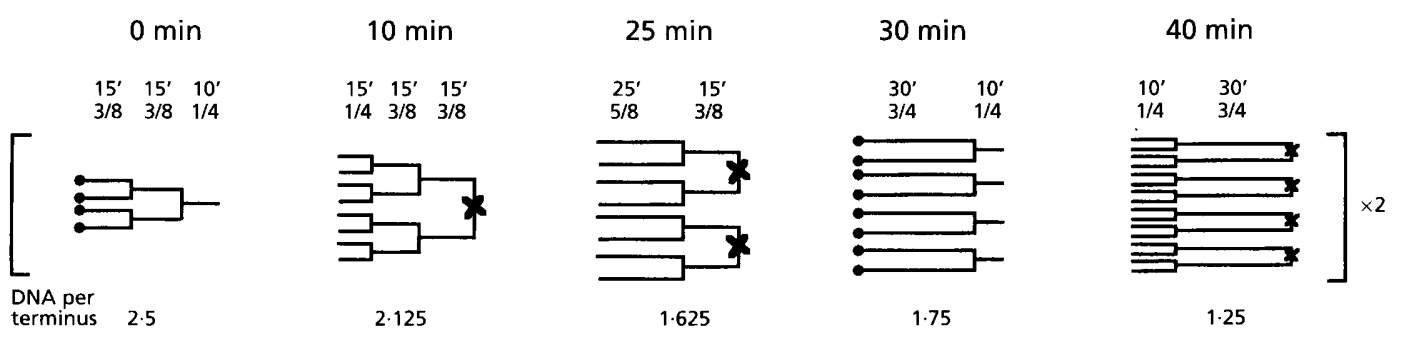

(c) After step-up $(C=40 \mathrm{~min})$
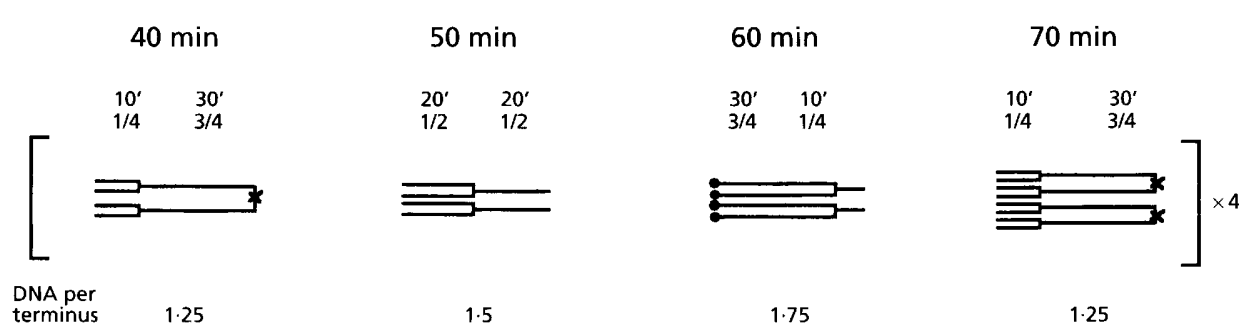

Fig. 1. Schematic representation of chromosome configurations in a cell with a doubling time of 30 min (a) during steady-state growth with a $C$ time of 80 min due to thymine limitation, (b) during transition to a faster chromosome replication caused by deoxyguanosine and thymine addition, and (c) during steady-state growth with a $C$ time of 40 min. Half chromosomes are depicted. Closed circles designate initiating oriC and crosses designate terminating terC. Replication forks move from left to right. Numbers below the indicated ages represent distances between origins, fork(s) and terminus as time for replication and as genome equivalents. In addition, the total amount of DNA per terminus is indicated in genome equivalents. See Rationale for details.

unpublished) to test the hypothesis that planes of successive divisions in E. coli are not necessarily parallel using the round mutant $p b p A(\mathrm{ts})$, we concluded that it is impossible to discriminate between the two alternatives when cells grow and divide on an agar surface. Likewise, in a recent study on the rodA ftsA double mutant (Begg \& Donachie, 1998) the dividing cell had been fully separated to two daughters before the subsequent (secondary) constrictions became visible. This makes it difficult to ascertain that no slippage of the divided cell has occurred, and to establish whether the alternating division planes are restricted to two dimensions as suggested by Begg \& Donachie (1998) or can occur in three dimensions. Division planes alternating in three dimensions were clearly observed by Bloom et al. (1974) in an $e n v B$ mutant, but could be related to its defective shape and peptidoglycan synthesis.
To discriminate between the two possibilities, parallel or alternating division planes, we developed a physiological method (see Rationale below) which induces cells that are otherwise not impaired in division to initiate secondary constrictions before separation at the primary division site. Secondary-constricted cells (SCCs) were obtained by thymine limitation of thy $A$ cultures (Pritchard \& Zaritsky, 1970). Simultaneous mecillinam treatment resulted in large spheroids with multi-forked replicating chromosomes (Helmstetter et al., 1968). Cells with several constrictions in different planes were indeed observed after removal of the drug following acceleration of the frequency of signals for successive divisions by adding deoxyguanosine (Beacham et al., 1971).

A preliminary version of the results given in this paper 
was presented at a workshop in Chorin, Germany (Zaritsky et al., 1997).

\section{RATIONALE}

To visualize secondary constrictions in a dividing cell, two conditions must be fulfilled: faster frequency of division signals and extended period of the division process. The duration $T$ of visible constriction is proportional to cell diameter and inversely related to the culture growth rate (Woldringh et al., 1988). Thus, $T$ will be longer in cells with larger girth at a particular nutritional state and temperature, obtained by mecillinam treatment, for example.

An obligatory signal for constriction-initiation to be enhanced is completion of a round of chromosome replication, which follows replication-initiation by $C$ min (the time required for a round to complete) (Helmstetter et al., 1968). In a balanced growth situation (Fishov et al., 1995), the frequency of terminations equals that of initiation events which, in turn, follow mass doublings (Pritchard et al., 1969). To modify this frequency without changing growth rate, one can manipulate $C$ by changing the concentration of external thymine supplied to thy A strains (Zaritsky \& Pritchard, 1973). When such cells, which grow on low thymine levels with slow-replicating chromosomes thus having several replication forks, are transferred to a high level of thymine ('stepped-up'; Pritchard \& Zaritsky, 1970), their existing forks proceed faster to terminate earlier and division rate is enhanced (Zaritsky, 1971).

As an example (resembling the principal experiment), half a schematic chromosome in a culture with doubling time $\tau$ of $30 \mathrm{~min}$ is depicted in Fig. 1, one that is initiating a new round of replication upon the transition. Its configurations are displayed at certain ages (measured from an initiation) when two discrete events occur in the cycle, initiation and termination, and once between them. The configurations during a transition between $C=80 \mathrm{~min}$ to $C=40 \mathrm{~min}$ are flanked by those in the pre- and post-step steady-states. Three terminations are seen to take place during the transition. For clarity purposes, the scheme includes distances between the origins, fork(s) and the terminus on the half-chromosomes described in terms of genome equivalents. The chromosome complexity, expressed by number of replication 'positions' $(n=C / \tau$; Sueoka \& Yoshikawa, 1965), is given as the amount of DNA per terminus (DNA/Ter) in genome equivalents (Bremer \& Dennis, 1996), below each configuration. The mean value of $n$ decreases from 2.67 to $1 \cdot 33$. DNA/Ter can be calculated by weighing the proportion of chromosomes in each age group (Powell, 1956). It can also be derived from the ratio between DNA/cell (Helmstetter et al., 1968) to Ter/cell (Zaritsky \& Pritchard, 1973), yielding DNA/Ter $=\tau \times\left(2^{C / \tau}-1\right) /(C \times \ln 2)$. The value of DNA/Ter gradually declines from 2.89 to 1.64 , the values calculated for the two respective steady states.

\section{METHODS}

Bacterial strains, growth media and measurements. E. coli $\mathrm{K}$ 12 strain CR34 (thr-1 leuB6 thyA) (Zaritsky \& Pritchard, 1971; Zaritsky \& Woldringh, 1978; Molina et al., 1998) was grown aerobically with a doubling time $\tau=30 \mathrm{~min}$ at $37^{\circ} \mathrm{C}$ in glucose minimal medium (Woldringh et al., 1994) supplemented with Casamino acids $(1 \%, \mathrm{w} / \mathrm{v} ;$ Difco $)$ and thymine as indicated. For fast replication, deoxyguanosine $(100 \mu \mathrm{g}$ $\mathrm{ml}^{-1}$; Sigma) was added (Beacham et al., 1971). The OD was determined with a spectrophotometer (LKB Ultraspec II), and cell concentration was measured with an electronic particle counter $(30 \mu \mathrm{m}$ orifice). Balanced cultures were grown 'normally' (Fishov et al., 1995) for at least 10 doublings by successive dilutions $\left(\mathrm{OD}_{450}<0 \cdot 4\right)$.

Secondary constrictions. Large CR 34 cells with multi-forked chromosomes were obtained by fast growth $(\tau \sim 30 \mathrm{~min})$ and slow replication $\left(C \sim 80 \mathrm{~min}\right.$ ) with $5 \mu \mathrm{g}$ thymine $\mathrm{ml}^{-1}$ (Zaritsky \& Pritchard, 1971; Molina et al., 1998). Spheroids were obtained by addition of mecillinam $\left(0 \cdot 8-1 \mu \mathrm{g} \mathrm{ml}^{-1}\right)$. The most suitable cells were obtained by removing the drug at 60-70 min by filtration (on polycarbonate Poretics filters with $0.4 \mu \mathrm{m}$ pores) (Spratt, 1978), and adding deoxyguanosine (to $100 \mu \mathrm{g} \mathrm{ml}^{-1}$ ) and thymine (to $15 \mu \mathrm{g} \mathrm{ml}^{-1}$ ) to enhance replication rate (Beacham et al., 1971; Zaritsky \& Woldringh, 1978) and to let the ongoing forks terminate more frequently, thus triggering successive divisions (Fig. 1). Quantitatively, doubling the rate of replication (from a round in $80 \mathrm{~min}$ to one in $40 \mathrm{~min}$ ) results in transiently halving the period between successive terminations from $\sim 30 \mathrm{~min}$ ( $\tau$-equivalent) to $\sim 15$ min. SCCs thus obtained were fixed with formaldehyde $(0.25 \%, \mathrm{v} / \mathrm{v})$ for analyses. The flow chart and accompanying pictures (Fig. 2) describe the overall scheme of this series of experiments.

Microscopical observations and analyses. Cells were fixed with formaldehyde $(0.25 \%)$ and stained with DAPI $\left(4^{\prime}, 6-\right.$ diamidino-2-phenylindole dihydrochloride hydrate $)(0 \cdot 2 \mu \mathrm{g}$
'Normal' growth in glucose minimal medium + Casamino acids with $5 \mu \mathrm{g}$ thymine $\mathrm{ml}^{-1}(C \sim 80 \mathrm{~min})$, to get large cells with multi-forked chromosomes

\section{$\downarrow$}

$60 \mathrm{~min}$ in mecillinam $\left(1 \mu \mathrm{g} \mathrm{ml}^{-1}\right)$ to get spheroidal cells $\downarrow$

Wash and resuspend in thymine $\left(15 \mu \mathrm{g} \mathrm{ml}^{-1}\right)$ and $2^{\prime}$-deoxyguanosine $\left(100 \mu \mathrm{g} \mathrm{ml}^{-1}\right)$ (C $40 \mathrm{~min})$

to enhance replication rate

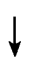

After $60 \mathrm{~min}$ in same medium all cells show constrictions and 'butterfly-like' shape
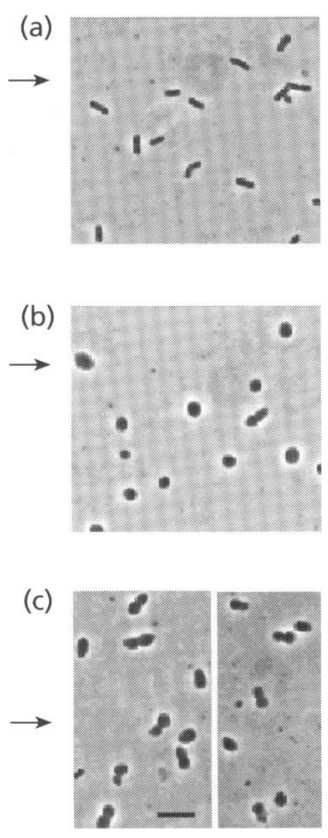

Fig. 2. Overall experimental scheme. Bar, $5 \mu \mathrm{m}$. 
$\mathrm{ml}^{-1}$ ) for at least $1 \mathrm{~h}$. The cells were immobilized on object slides coated with a dried $2 \%(\mathrm{w} / \mathrm{v})$ agarose layer. The preparations were illuminated at $330-380 \mathrm{~nm}$. Images were taken with an air-cooled charged-coupled-device (CCD) camera (Princeton Instruments; model RTE-1317-k-1), mounted on an Olympus $\mathrm{BH}-2$ fluorescence microscope equipped with an oil immersion $(\times 100)$ lens, a $5 \times$ photoocular and an emission filter of $420 \mathrm{~nm}$. A computer (Quadra $840 \mathrm{AV}$; Apple) was used for image collection and exposure control via mechanical lamp and CCD camera shutters.

For three-dimensional visualization of SCCs, the cells were stained with FM 4-64 (catalogue no. T-3166; Molecular Probes) $\left(2 \mu \mathrm{g} \mathrm{ml}^{-1}\right.$ final concentration) and immersed in $2 \%$ (w/v) agarose (Vida \& Emr, 1995). Images were collected by confocal scanning laser microscopy (CSLM), using a Leica microscope equipped with a $488 / 514$ dual-band argon-ion laser and an oil-immersion objective $(\times 100$, n.a. $=1 \cdot 32)$. For scanning electron microscopy ( $\mathrm{SEM}$ ), $\mathrm{OsO}_{4}$-fixed cells were dehydrated in alcohol, deposited on a nuclear pore filter and critical-point-dried.

Image processing and analysis, length and diameter of cells and filaments, number of nucleoids, and position and number of constriction sites were all measured using the package Object-Image version (Vischer et al., 1994) of NIH-image software.

\section{RESULTS}

\section{Multi-nucleoid, spheroidal cells}

Thymine-limited thy A cells of E. coli K-12 CR34 are larger than the wild-type due to delayed divisions at slow replication rates (Zaritsky \& Woldringh, 1978). The additional mass is accommodated by expansion in both dimensions, length and diameter (Zaritsky \& Pritchard, 1973; Zaritsky, 1975; Woldringh et al., 1994). The extended time $(T)$ required to complete a division of such fatter cells results in increased amount of DNA per cell, as seen in the examples of Fig. 3(a) (and see Zaritsky \& Woldringh, 1978). Moving the focus under the microscope revealed the abundant amount of (DAPIstained) DNA to be located mostly in the cell periphery (not shown). The proportionality between DAPI fluorescence and amount of DNA in bacterial nucleoids has been confirmed by image cytometry (N. O.E. Vischer, P. G. Huls, R. I. Ghauharali, G. J. Brakenhoff \& C. L. Woldringh, unpublished).

Such 'normally' grown (Fishov et al., 1995) cylindrical cells gradually transform to spheroids when treated with mecillinam (Spratt, 1978). In the large, spheroidal cells, DNA was also found exclusively near the periphery (Fig. 3b; and see Fig. 2 of Normark, 1969).

\section{Secondary-constricted cells}

Several schedules were employed to find out the optimal conditions to harvest a high proportion of SCCs. The best results were obtained by washing off mecillinam 60-70 min (approx. 2 $\tau$ ) after its addition and resuspending them in the same medium but with deoxyguanosine and thymine (Fig. 2). This addition temporarily triggered divisions more frequently due to enhancement of chromosome replication rate ('step-
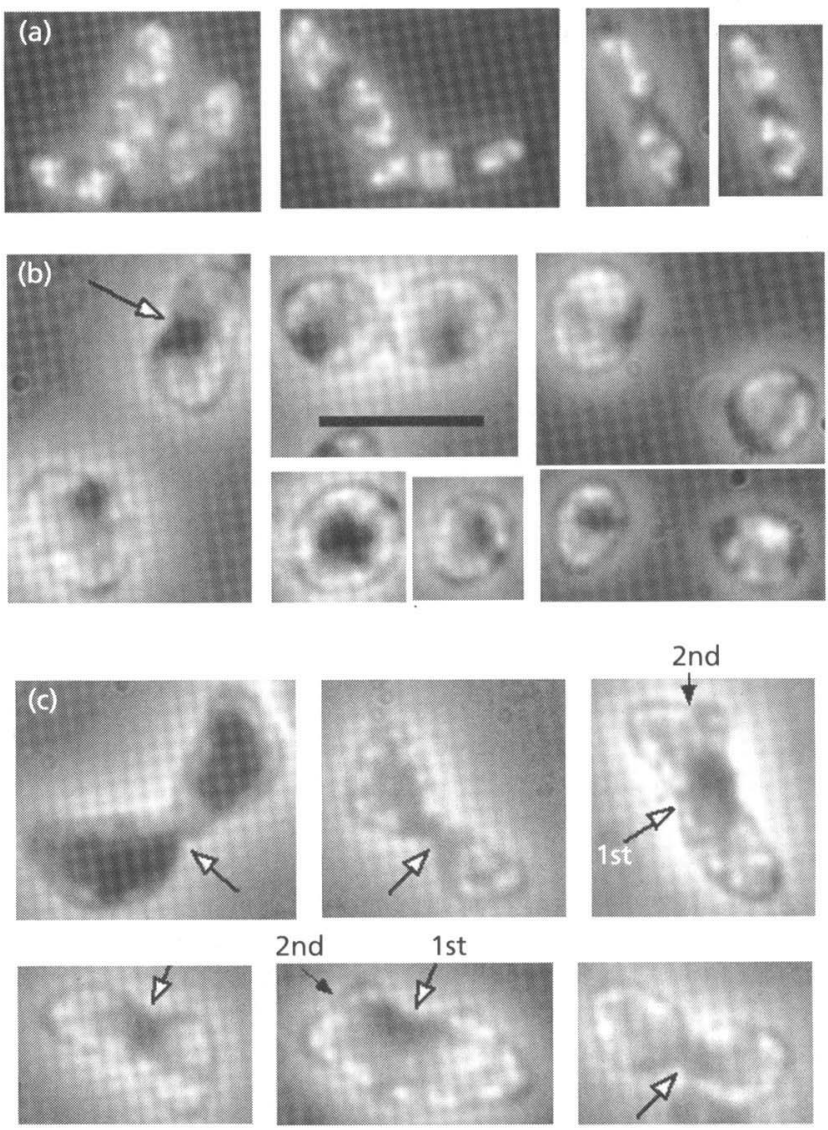

Fig. 3. Fluorescence microscopy of the three populations of DAPI-stained cells indicated in Fig. 2(a-c). White arrows indicate primary constrictions and black arrows indicate secondary constrictions. Bar, $2 \mu \mathrm{m}$.

up'; Fig. 1). The large, ovoid cells in the samples drawn during the next $40 \mathrm{~min}$ had similar shapes: they were dividing, and many exhibited multiple constrictions and protruberances in various planes (Fig. 3c). Over $60 \%$ showed 'butterfly-like' shapes, presumably resulting from asymmetric constrictions (arrows in Fig. 3c; and see Discussion). This figure validates the approach described above (Rationale) to achieve partial synchrony of division, and is concordant with accelarated divisions following a step-up in thymine concentration (Zaritsky \& Pritchard, 1971).

\section{Three-dimensional observations}

Two scanning techniques were used to find out the planes of surface invaginations marking divisions, SEM and CSLM. Two typical examples of SCCs are displayed in Fig. 4, each photographed by SEM after tilting it over the maximum possible range $\left(55^{\circ}\right)$. In both, two secondary constrictions are seen to lie perpendicularly to the primary division plane. Tertiary constrictions were occasionally traced, but this method is not sufficiently sensitive to detect their exact locations or planes. 


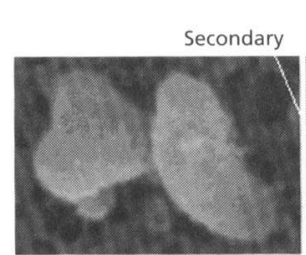

$-30^{\circ}$

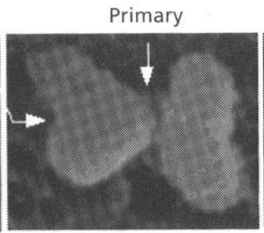

Top view $\left(0^{\circ}\right)$

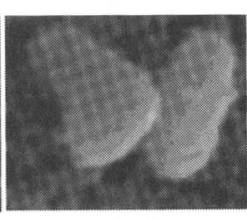

$+25^{\circ}$

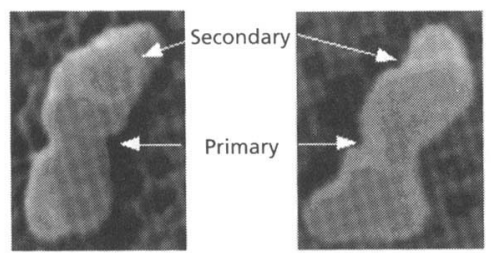

Two views tilted $55^{\circ}$

Fig. 4. Scanning electron micrographs of SCCs. Two representative cells from Fig. 3(c) were photographed from different angles, as indicated. Arrows indicate sites of primary and secondary constrictions. Magnification $\times 10000$.
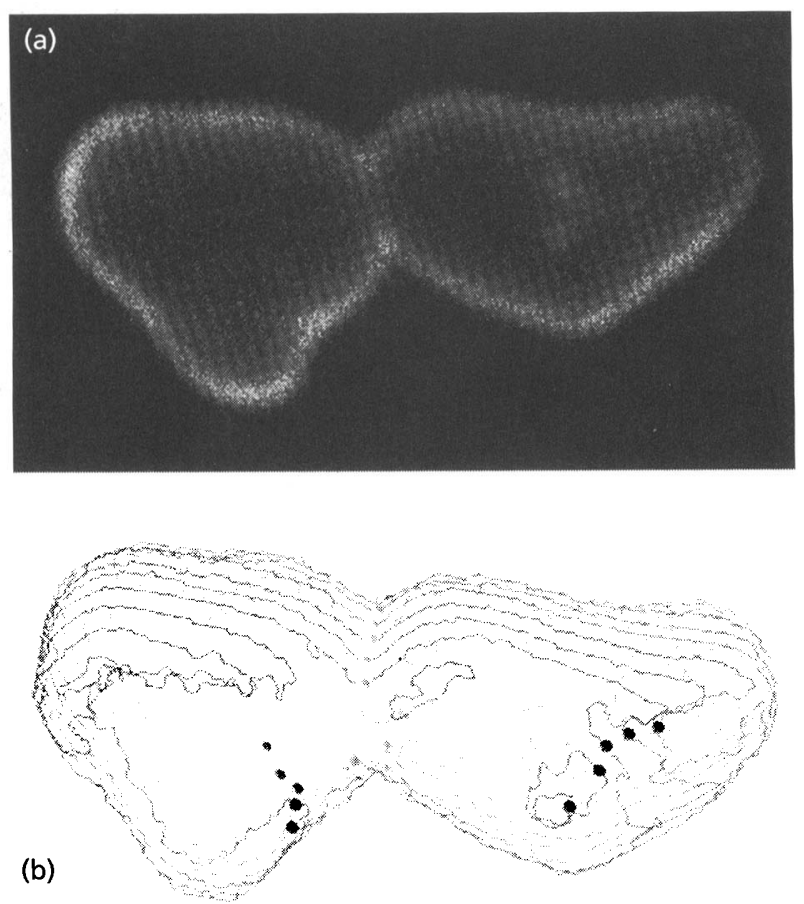

Fig. 5. Confocal scanning laser micrograph of a representative cell from Fig. 3(c) stained with FM 4-64. (a) Computergenerated projection and (b) its three-dimensional reconstruction as 'wire-frames'. The balls were placed independently on the centres of clear invaginations in each slice; their appearance in one plane in the three-dimensional picture was interpreted as a constriction. Primary (grey balls) and secondary (black balls) constrictions are labelled.

CSLM was exploited to scan $0 \cdot 2-0 \cdot 3 \mu \mathrm{m}$ slices of SCCs labelled with the membrane-specific fluorochrome FM 4-64 (Vida \& Emr, 1995). The resultant stacks of tracings from such cells were reconstructed. A projection and two different views of a typical cell are displayed in Fig. 5, which shows secondary constrictions in planes oblique to that of the primary. The asymmetric, 'butterfly-like' shape found in the majority of cells (Figs $3 c, 4,5$ ), is caused by secondary constrictions that start more or less perpendicular to the primary constriction and to each other. In other words, division planes can occur in three dimensions and are not necessarily restricted to one (as suggested by Cooper, 1997) or two (as suggested by Begg \& Donachie, 1998) dimensions.

An electronic/digital version of this cell, which can be moved around, exists in our web pages at: http://simon.bio.uva.nl/multiconstrictedcell.html (or http://www.bgu.ac.il/life/zaritsky.html). It can best be viewed using Netscape 4.05 and Quicktime 2.5.

\section{DISCUSSION}

We have developed a method to generate dividing E. coli cells that initiate secondary constrictions before separation by (i) modifying cell shape with mecillinam and (ii) accelerating terminations with a thymine step-up. In contrast to most previous studies, cells were grown and fixed in suspension before preparation for microscopy. Dividing cells adopted butterfly-like shapes, often displaying secondary constrictions, as previously seen in an envB mutant (Figs 5 and 12 in Bloom et al., 1974). The results demonstrate that planes of successive divisions are not parallel as observed by Cooper (1997) for $\operatorname{rodA}$ cells growing in Methocel, neither are they precisely perpendicular as observed by Donachie et al. (1995) and Begg \& Donachie (1998) for rodA fts A cells growing on agar. Although our conditions were somewhat different, the simplest explanation for this phenomenon is that successive division planes are more or less perpendicular to each other, and tilted in other directions depending on the positions of segregating nucleoids and the development of new growth axes. In a spherical cell too, segregating DNA represents a bipolar structure (e.g. Niki \& Hiraga, 1998), which eventually creates a longitudinal growth axis and elliptical cell shape. This is represented schematically in Fig. 6 for successive divisions in rod-shaped and spherical cells growing on agar, in Methocel or in liquid suspension.

When cylindrical cells (Fig. 6a), dividing in a perpendicular plane to the long axis, are grown on an agar surface, daughters are not free to continue elongation but are pushed together after slipping out of alignment with each other (Donachie \& Begg, 1970; Mulder \& Woldringh, 1993). In the less solid Methocel, divided cells stay in line (Fig. 6a), thus forming a chain oriented in a straight path (see Fig. 1n-p of Cooper, 1997).

Spheroidal cells usually develop asymmetric constrictions (Bloom et al., 1974; Iwaya et al., 1978; Normark, 1969), presumably because of their larger diameter. As growth continues, two new longitudinal axes develop in the prospective daughter cells, which may become tilted depending on the degree of asymmetry of the constriction. When grown on agar, the daughters are kept together, forming tetrads after the next division. Similar tetrads have been found in agargrown Neisseria gonorrhoeae (Westling-Haggström et 
(a) Cylindrical cell

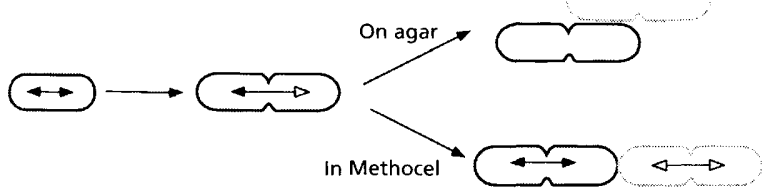

(b) Spherical cell

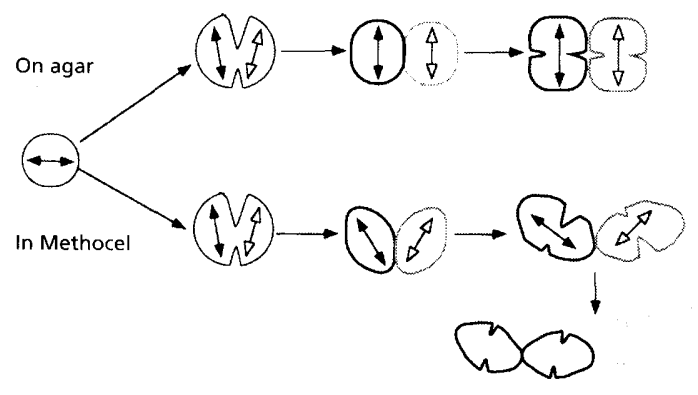

(c) Development of 'butterfly-like' cell

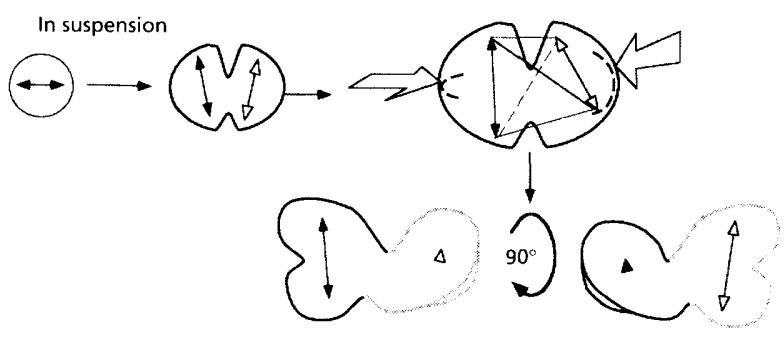

Fig. 6. Schematic representation of (a) cylindrical and (b) spherical cells growing on an agar surface or in Methocel, and (c) the development of a 'butterfly-like' cell. Double-headed arrows indicate hypothetical bipolar nucleoid structures generating longitudinal growth axes. The butterfly-like shape, drawn in two positions turned $90^{\circ}$ to one another (cf. Figs $3 c$, 4,5 ), is proposed to develop via an orientation of the growth axes in such a way that their ends lie in the apexes of a tetrahedron, as detailed in Discussion. The cells or half-cells with different colours (black and grey) and arrows (closed and open) developed from two cell halves of the preceding division.

al., 1977). In Methocel, the divided cells become oriented in a wriggly path (Fig. 6b) because they develop asymmetric constrictions and tilted growth axes and are not kept together in the less solid medium. In such chains the division planes only seem parallel, as interpreted by Cooper (1997). A similar conclusion to ours was reached by Begg \& Donachie (1998).

The present spheroidal, relatively large cells (Figs 3c, 4, 5) were obtained by mecillinam treatment and thymine limitation. Mass growth of several E. coli thy A strains, including CR34, continues indefinitely during thymine limitation (Zaritsky \& Pritchard, 1971). At fast growth rates $(\tau<\sim 50 \mathrm{~min})$, cell size rises gradually in both dimensions, length and diameter (Zaritsky \& Pritchard, 1973). Increased cell girth, in turn, results in a longer time $(T)$ to complete a constriction leading to division (Woldringh et al., 1988), and thus in multi-nucleated cells (Fig. 4a). This is one condition necessary to obtain secondary constrictions (see Rationale). The second condition, more frequent terminations of replication rounds to initiate constrictions, was achieved by accelerating replication rate upon raising external thymine concentration (Pritchard \& Zaritsky, 1970) and confirmed by acceleration of divisions (Zaritsky, 1971). As emphasized by Donachie et al. (1995), spheroidal cells become multichromosomal due to delayed divisions, but constrictions still initiate between segregated nucleoids and reflect their positions (Fig. 3). A similar delay in division was observed in populations of $p b p A$ (Woldringh et al., 1988) and envB (Bloom et al., 1974) mutants. It must also have occurred in the spheroidal, thymine-limited cells described here (Fig. $2 \mathrm{~b})$, as indicated by the many, large nucleoids present (Fig. 3b).

The continued growth and delayed division allow the two new longitudinal growth axes generated by the primary constriction to become maximally tilted. The driving force for such a movement may be supplied by spatial constraints that become fully relieved when segregating nucleoids become located in the apexes of a regular tetrahedron (like four identical atoms in the molecule $\mathrm{P}_{4}$ ), possibly corresponding to minimal energy of interaction (among themselves or with other cellular components) and optimal space occupation (see schematic representation in Fig. 6c). Such a configuration implies that the new growth axes are perpendicular to the first and to each other (in three dimensions), and that the secondary division planes (perpendicular to these axes) are perpendicular to each other as well as to the primary division plane between the two regions of nucleoids. The lower degree of symmetry here is due to a higher amount of DNA per cell, lack of ideal synchrony in terminations and unaccomplished segregations (Fig. $3 c)$.

Many Y-shaped cells in the treated populations (Fig. 4) are reminiscent of branched cells, previously observed (e.g. Zaritsky, 1977; Iwaya et al., 1978; Akerlund et al., 1993; Henderson et al., 1997) but without understanding their origin. Similar cells have been obtained by slowing down the growth rate (nutritional shift-down) during step-up of the replication rate (Woldringh et al., 1994, 1995), but the straight-jacketed cylindrical peptidoglycan often did not allow the segregating nucleoids to move freely about. Spheroids, which presumably relieve the spatial constraint, were created here by blocking $\mathrm{Pbp} 2$ with mecillinam. The observed bifurcation of the cell pole implies a perpendicular division plane.

Cooper (1997) has suggested that, if successive division planes were oriented at right angles, a major and unlikely reorganization of the peptidoglycan chains would be required since a cell grows by inserting new 'hoops' of peptidoglycan between existing hoops, which run circumferentially, perpendicular to its long axis. However, the chains are very short (a mean length of 5-10 $\mathrm{nm})$ compared to cell circumference $(2500 \mathrm{~nm})$, and are not necessarily oriented parallel to each other (Höltje, 1998). Consequently, changes in the shape of the sacculus do not require reorientation of the chains. Such changes indeed occur upon nutritional shifts (Zaritsky 
et al., 1982, 1993); a cell is thus able to change the orientation of its division plane within one or two generations.

\section{ACKNOWLEDGEMENTS}

This work was partially supported by an EMBO short-term fellowship (award ASTF 8548) to I. F. Wijnand Takkenberg is gratefully acknowledged for technical help, particularly with SEM. An anonymous referee is gratefully acknowledged for helpful remarks, which clarified our ideas.

\section{REFERENCES}

Åkerlund, T., Nordström, K. \& Bernander, R. (1993). Branched Escherichia coli cells. Mol Microbiol 10, 849-858.

Beacham, I. R., Beacham, K., Zaritsky, A. \& Pritchard, R. H. (1971). Intracellular thymidine triphosphate concentrations in wild type and thymine requiring mutants of Escherichia coli 15 and K12.J Mol Biol 60, 75-86.

Begg, K. J. \& Donachie, W. D. (1998). Division planes alternate in spherical cells of Escherichia coli. J Bacteriol 180, 2564-2567.

Bernander, R. \& Nordström, K. (1990). Chromosome replication does not trigger cell division in E. coli. Cell 60, 365-374.

Bloom, G. D., Gumpert, J., Normark, S., Schuhmann, E., Taubeneck, U. \& Westling, B. (1974). Morphology and growth pattern of a rod-negative envB mutant of Escherichia coli K12. Z Allg Mikrobiol 14, 465-477.

Bouloc, P., Jaffe, A. \& D'Ari, R. (1989). The Escherichia coli lov gene product connects peptidoglycan synthesis, ribosomes and growth rate. $E M B O J \mathbf{8}, 317-323$.

Bremer, H. \& Dennis, P.P. (1996). Modulation of chemical composition and other parameters of the cell by growth rate. In Escherichia coli and Salmonella: Cellular and Molecular Biology, 2nd edn, pp. 1553-1569. Edited by F. C. Neidhardt and others. Wäshington, DC: American Society for Microbiology.

Cooper, S. (1991). Bacterial Growth and Division: Biochemistry and Regulation of Prokaryotic and Eukaryotic Division Cycles. San Diego: Academic Press.

Cooper, S. (1997). Division pattern of a round mutant of Escherichia coli. J Bacteriol 179, 5582-5584.

D'Ari, R. \& Bouloc, P. (1990). Logic of the Escherichia coli cell cycle. Trends Biochem Sci 15, 191-194.

Donachie, W. D. \& Begg, K. J. (1970). Growth of the bacterial cell. Nature 227, 1220-1224.

Donachie, W. D., Addinall, S. \& Begg, K. J. (1995). Cell shape and chromosome partition in prokaryotes or, why $E$. coli is rodshaped and haploid. Bioessays 17, 569-576.

Fishov, I., Zaritsky, A. \& Grover, N. B. (1995). On microbial states of growth. Mol Microbiol 15, 789-794.

Hadas, H., Einav, M., Fishov, I. \& Zaritsky, A. (1995). Divisioninhibition capacity of penicillin in Escherichia coli is growth-rate dependent. Microbiology 141, 1081-1083.

Helmstetter, C. E., Cooper, S., Pierucci, O. \& Revelas, L. (1968). The bacterial life sequence. Cold Spring Harbor Symp Quant Biol 33, 807-822.

Henderson, T. A., Young, K. D., Denome, S. A. \& Elf, P. K. (1997). $\mathrm{AmpC}$ and $\mathrm{AmpH}$, proteins related to the class $\mathrm{C} \beta$-lactamases, bind penicillin and contribute to the normal morphology of Escherichia coli. J Bacteriol 179, 6112-6121.

Höltje, J.-V. (1998). Growth of the stress-bearing and shape- maintaining murein sacculus of Escherichia coli. Microbiol Mol Biol Rev 62, 181-203.

Iwaya, M., Goldman, R., Tipper, D. J., Feingold, B. \& Strominger, J. L. (1978). Morphology of an Escherichia coli mutant with a temperature-dependent round cell shape. J Bacteriol 136, $1143-1158$

Lane, H. E. D. \& Denhardt, D. T. (1975). The rep mutation. IV. Slower movement of replication forks in Escherichia coli rep strains. J Mol Biol 97, 99-112.

Mitchison, J. M. (1971). The Biology of the Cell Cycle. Cambridge, UK: Cambridge University Press.

Molina, F., Jiménez-Sánchez, A. \& Guzmán, E. C. (1998). Determining the optimal thymidine concentration for growing Thy ${ }^{-}$Escherichia coli strains. J Bacteriol 180, 2992-2994.

Mulder, E. \& Woldringh, C. L. (1993). Plasmolysis bays in Escherichia coli: are they related to development and positioning of division sites? J Bacteriol 175, 2241-2247.

Niki, H. \& Hiraga, S. (1998). Polar localization of the replication origin and terminus in Escherichia coli nucleoids during chromosome partitioning. Genes Dev 12, 1036-1045.

Normark, S. (1969). Mutation in Escherichia coli K-12 mediating spherelike envelopes and changed tolerance to ultraviolet irradiation and some antibiotics. J Bacteriol 98, 1274-1277.

Powell, E. O. (1956). Growth rate and generation time of bacteria, with special reference to continuous culture. J Gen Microbiol 15, $492-511$.

Pritchard, R. H. \& Zaritsky, A. (1970). Effect of thymine concentration on the replication velocity of DNA in a thymineless mutant of Escherichia coli. Nature 226, 126-131.

Pritchard, R. H., Barth, P. T. \& Collins, J. (1969). Control of DNA synthesis in bacteria. Symp Soc Gen Microbiol 19, 263-297.

Spratt, B. G. (1978). Escherichia coli resistance to $\beta$-lactam antibiotics through a decrease in the affinity of a target for lethality. Nature 274, 713-715.

Sueoka, N. \& Yoshikawa, Y. (1965). The chromosome of Bacillus subtilis. I. The theory of marker frequency analysis. Genetics $\mathbf{5 2}$, 747-757.

Van Helvoort, J. M. L. M., Huls, P. G., Vischer, N. O. E. \& Woldringh, C. L. (1998). Fused nucleoids resegregate faster than cell elongation in Escherichia coli $p b p B(\mathrm{Ts})$ filaments after release from chloramphenicol inhibition. Microbiology 144, 1309-1317.

Vida, T. A. \& Emr, S. D. (1995). A new vital stain for visualizing vacuolar membrane dynamics and endocytosis in yeast. $J$ Cell Biol 128, 779-792.

Vischer, N. O. E., Huls, P. G. \& Woldringh, C. L. (1994). Objectimage: an interactive image analysis program using structured point collection. Binary 6, 160-166.

Westling-Haggström, B., Elmros, T., Normark, S. \& Winblad, B. (1977). Growth pattern and cell division in Neisseria gonorrhoeae. $J$ Bacteriol 129, 333-342.

Woldringh, C. L., Huls, P. G., Nanninga, N., Pas, E., Taschner, P. E. M. \& Wientjes, F. B. (1988). Autoradiographic analysis of peptidoglycan synthesis in shape and cell division mutants of Escherichia coli MC4100. In Antibiotic Inbibition of Bacterial Cell Surface Assembly and Function, pp. 66-78. Edited by P. Actor and others. Washington, DC: American Society for Microbiology.

Woldringh, C. L., Zaritsky, A. \& Grover, N. B. (1994). Nucleoid partitioning and the division plane in Escherichia coli.J Bacteriol 176, 6030-6038.

Woldringh, C. L., Zaritsky, A. \& Grover, N. B. (1995). Nucleoid 
partitioning by segregation axes in Escherichia coli. In Keystone Symposium on Molecular and Cellular Biology, Santa Fe, New Mexico, abstract A2-248, p. 121 (J Cell Biochem suppl. 19A). New York: Wiley.

Zaritsky, A. (1971). Studies on DNA replication and cell division in bacteria, pp. 50-57. PhD thesis, University of Leicester.

Zaritsky, A. (1975). On dimensional determination of rod-shaped bacteria. J Theor Biol 54, 243-248.

Zaritsky, A. (1977). Branching of fast-growing Escherichia coli $15 \mathrm{~T}$ at low thymine concentrations. FEMS Microbiol Lett 2, 65-69.

Zaritsky, A. \& Pritchard, R. H. (1971). Replication time of the chromosome in thymineless mutants of Escherichia coli. J Mol Biol 60, 65-74.

Zaritsky, A. \& Pritchard, R. H. (1973). Changes in cell size and shape associated with changes in the replication time of the chromosome of Escherichia coli. J Bacteriol 114, 824-837.
Zaritsky, A. \& Woldringh, C. L. (1978). Chromosome replication rate and cell shape in Escherichia coli: lack of coupling. $J$ Bacteriol 135, 581-587.

Zaritsky, A., Grover, N. B., Naaman, J., Woldringh, C. L. \& Rosenberger, R. F. (1982). Growth and form in bacteria. Comments Mol Cell Biophys 1, 237-260.

Zaritsky, A., Woldringh, C. L., Helmstetter, C. E. \& Grover, N. B. (1993). Dimensional rearrangement of Escherichia coli $\mathrm{B} / \mathrm{r}$ cells during a nutritional shift-down. J Gen Microbiol 139, 2711-2714.

Zaritsky, A., Woldringh, C. L., Fishov, I., Van Geel, A. \& Einav, M. (1997). Multi-constricted Escherichia coli cells with varying division planes. In The Bacterial Cell Cycle Workshop, Chorin, Germany, September 13-17 1997, abstract P43. Edited by W. Messer \& J.-V. Höltje. Berlin: MPI für molekulare Genetik.

Received 12 October 1998; revised 2 January 1999; accepted 14 January 1999. 